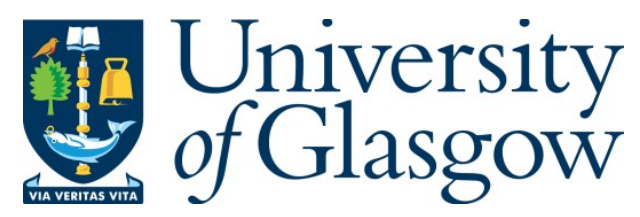

$\mathrm{Ng}, \mathrm{F}$. C. et al. (2021) Cerebral edema in patients with large hemispheric infarct undergoing reperfusion treatment: a HERMES meta-analysis. Stroke, 52(11), pp. 34503458 .

(doi: 10.1161/STROKEAHA.120.033246)

This is the Author Accepted Manuscript.

There may be differences between this version and the published version. You are advised to consult the publisher's version if you wish to cite from it.

https://eprints.gla.ac.uk/240881/

Deposited on: 14 May 2021

Enlighten - Research publications by members of the University of Glasgow http://eprints.gla.ac.uk 


\section{Cerebral edema in patients with large hemispheric infarct undergoing reperfusion}

\section{treatment: a HERMES meta-analysis}

Felix C. Ng MBBS ${ }^{1,2}$, Nawaf Yassi PhD ${ }^{1,3}$, Gagan Sharma MCA ${ }^{1}$, Scott B. Brown PhD Mayank Goyal MD ${ }^{5}$ Charles BLM Majoie MD6, Tudor G. Jovin MD7, Michael D. Hill MD ${ }^{8}$, Keith W. Muir MD ${ }^{9}$, Jeffrey L. Saver MD ${ }^{10}$, Francis Guillemin $\mathrm{PhD}^{11}$, Andrew M. Demchuk $\mathrm{MD}^{8}$, Bijoy K. Menon MD ${ }^{8}$, Luis San Roman MD ${ }^{12}$, David S. Liebeskind MD ${ }^{13}$, Philip White $\mathrm{MD}^{14}$, Diederik W.J. Dippel MD ${ }^{15}$, Antoni Davalos $\mathrm{MD}^{16}$, Serge Bracard MD ${ }^{17}$, Peter J. Mitchell MMed $^{18}$, Michael J. Wald $\mathrm{PhD}^{19}$, Stephen M Davis MD ${ }^{1}$, Kevin N. Sheth $\mathrm{MD}^{20}$, W. Taylor Kimberly $\mathrm{MD}^{21^{*}}$, Bruce C.V. Campbell $\mathrm{PhD}^{1^{*}}$, for the HERMES collaborators

*contributed equally as joint senior authors

1. Department of Medicine and Neurology, Melbourne Brain Centre at the Royal Melbourne Hospital, University of Melbourne, Parkville, Australia

2. Department of Neurology, Austin Health, Heidelberg, Australia

3. Population Health and Immunity Division. The Walter and Eliza Hall Institute of Medical Research. Parkville, Australia.

4. Altair Biostatistics, St Louis Park, Minnesota, USA

5. Department of Radiology, University of Calgary, Foothills Hospital, Calgary, AB, Canada

6. Department of Radiology and Nuclear Medicine, Amsterdam University Medical Centers, location AMC, Amsterdam, the Netherlands

7. Cooper Neurological Institute, Cooper University Health Care, Camden, New Jersey, USA

8. Department of Clinical Neurosciences, Hotchkiss Brain Institute, Cumming School of Medicine, University of Calgary, Foothills Hospital, Calgary AB, Canada 
9. Institute of Neuroscience \& Psychology, University of Glasgow, Queen Elizabeth University

Hospital, Glasgow, UK

10. Department of Neurology and Comprehensive Stroke Center, David Geffen School of

Medicine at the University of California, Los Angeles, Los Angeles, California Stanford

Stroke Center, Stanford University, Stanford, California

11. Clinical Investigation Centre-Clinical Epidemiology INSERM 1433, University of

Lorraine and University Hospital of Nancy, Nancy, France

12. Department of Radiology, Hospital Clínic, Barcelona, Spain

13. Neurovascular Imaging Research Core, Department of Neurology, University of

California at Los Angeles, Los Angeles, California, USA

14. Institute of Neuroscience, Newcastle University, Newcastle upon Tyne, UK

15. Department of Neurology, Erasmus MC University Medical Center, Rotterdam, the Netherlands

16. Department of Neuroscience, Hospital Germans Trias i Pujol, Universitat Autònoma de Barcelona, Barcelona, Spain

17. Department of Diagnostic and Interventional Neuroradiology, INSERM U 947, University of Lorraine and University Hospital of Nancy, Nancy, France

18. Department of Radiology, Royal Melbourne Hospital, University of Melbourne, Parkville, Australia

19. Biogen, Cambridge, Massachusetts, USA

20. Department of Neurology, Yale New Haven Hospital, New Haven, Connecticut

21. Centre for Genomic Medicine and Department of Neurology, Massachusetts General Hospital, Boston 
Corresponding Author:

Bruce Campbell, bruce.campbell@mh.org.au

Department of Neurology, Royal Melbourne Hospital, Parkville 3050, Australia

Tel:+61393428448

Cover title: Cerebral edema in large infarction after EVT

Word:6078

References-29;Table-1;Figures-2

Key words: Infarction;Edema;Thrombectomy 


\section{$\underline{\text { ABSTRACT }}$}

Background and Purpose: Whether reperfusion into infarcted tissue exacerbates cerebral edema has treatment implications in patients presenting with extensive irreversible injury. We investigated the effects of endovascular thrombectomy and reperfusion on cerebral edema in patients presenting with radiological evidence of Large Hemispheric Infarction (LHI) at baseline

Methods: In a systematic review and individual patient-level meta-analysis of seven randomized controlled trials comparing thrombectomy versus medical therapy in anterior circulation ischemic stroke published between Jan 1, 2010 and May 31, 2017 (Highly Effective Reperfusion using Multiple Endovascular Devices (HERMES) collaboration), we analyzed the association between thrombectomy and reperfusion with maximal midline shift (MLS) on follow-up imaging as a measure of the space-occupying effect of cerebral edema in patients with LHI on pretreatment imaging, defined as diffusion-MRI or CT-perfusion ischemic core $80-300 \mathrm{~mL}$ or noncontrast CT-Alberta-Stroke-Program-Early-CT-Score (CT-ASPECTS) $\leq 5$. Risk of bias was assessed using the Cochrane tool.

Results: Among 1764 patients, 177 presented with LHI. Thrombectomy and reperfusion were associated with functional improvement (Thrombectomy common odds ratio $[\mathrm{cOR}]=2.30[95 \% \mathrm{CI}$ $1.32,4.00]$; reperfusion $\mathrm{cOR}=4.73[95 \% \mathrm{CI} 1.66,13.52]$ ) but not MLS (Thrombectomy $\beta=-$ $0.27[95 \%$ CI $-1.52,0.98]$; reperfusion $\beta=-0.78[95 \%$ CI $-3.07,1.50]$ ) when adjusting for age, NIHSS, glucose, and time-to-follow-up imaging. In an exploratory analysis of patients presenting with core volume $>130 \mathrm{~mL}$ or CT-ASPECTS $\leq 3(\mathrm{n}=76)$, thrombectomy was associated with greater MLS after adjusting for age and NIHSS ( $\beta=2.76[95 \%$ CI $0.33,5.20])$ but not functional improvement (OR 1.71[95\%CI 0.24,12.08]). 
Conclusion: In patients presenting with LHI, thrombectomy and reperfusion were not associated with MLS, except in the subgroup with very large core volume (>130ml) in whom thrombectomy was associated with increased MLS due to space-occupying ischemic edema. Mitigating cerebral edema-mediated secondary injury in patients with very large infarcts may further improve outcomes after reperfusion therapies. 
$\underline{\text { Non-Standard Abbreviations and Acronyms }}$

EVT Endovascular Thrombectomy

LHI Large Hemispheric Infarction

LOESS Locally Estimated Scatterplot Smoothing

MLS Midline Shift

NWU Net Water Uptake 


\section{Introduction}

Despite the well-established benefit of endovascular thrombectomy (EVT) in anterior circulation stroke with large vessel occlusion, over half of patients treated with EVT remain functionally dependent at follow-up. ${ }^{1}$ There is increasing focus on understanding the reasons for poor outcome and factors that limit the efficacy of EVT.

Cerebral edema, a recognized cause of secondary injury following ischemic stroke, is associated with substantial morbidity and mortality. ${ }^{2}$ Patients with large hemispheric infarction (LHI), defined as extensive ischemic stroke affecting the majority of the middle cerebral artery territory, ${ }^{3}$ are particularly prone to developing clinically significant brain swelling. More than $50 \%$ of these patients develop malignant cerebral edema and the mortality rate is up to $80 \%$ if untreated. ${ }^{4}$

The relationship between reperfusion and cerebral edema remains unclear. In animal models, reperfusion consistently exacerbates cerebral edema. However, clinical studies have suggested reduced edema after reperfusion. ${ }^{5-10}$ These conflicting results may indicate a variable response of cerebral tissue to reperfusion in which reperfusion may be detrimental in established infarction but protective via reduced infarct growth in salvageable tissue. ${ }^{5}$

Accordingly, patients presenting with LHI may be at particular risk of cerebral edema when treated with EVT. Many institutions do not restrict EVT based on the extent of pre-treatment ischemic changes, as supported by the available data. ${ }^{1,11}$ Understanding how treatment and reperfusion modulate cerebral edema in these patients is an increasingly relevant clinical consideration.

To date, evidence regarding the relationship between EVT and cerebral edema in the LHI subpopulation is scarce. In a patient-level meta-analysis of randomized controlled EVT trials, we 
investigate the effects of EVT and reperfusion on cerebral edema and the impact of cerebral edema on functional outcome in patients presenting with LHI.

\section{$\underline{\text { Methods }}$}

The anonymized individual patient data from the components trials are available on submission of an approved request to the VISTA-Endovascular data repository.

\section{Search strategy and selection criteria}

We performed a systematic review and meta-analysis according to PRISMA guidelines of randomized controlled trials comparing EVT with stent retrievers versus non-endovascular medical therapy in patients with anterior circulation ischemic stroke published between Jan 1, 2010 and May 31, 2017 ${ }^{1,11}$. Seven randomized controlled trials (MR-CLEAN, ${ }^{12}$ EXTEND-IA, ${ }^{13}$ ESCAPE,${ }^{14}$ SWIFT PRIME,${ }^{15}$ REVASCAT,${ }^{16}$ PISTE,${ }^{17}$ and THRACE $^{18}$ were identified using the search string ((“'randomized controlled trial”[Publication Type]) AND ((thrombectomy[Title/Abstract]) OR (clot retrieval[Title/Abstract]) OR intraarterial[Title/Abstract]) AND (stroke[Title/Abstract])) on Pubmed. Individual patient-level data were pooled by the Highly Effective Reperfusion using Multiple Endovascular Devices (HERMES) collaboration. We report our findings according to the PRISMA-IPD checklist and provide a flow diagram for studies and data inclusion. (Supplemental Material). The metaanalysis was prospectively designed by the HERMES executive committee, but not registered. The details of the HERMES collaboration initiation, methodology of meta-analysis search strategy, individual participant data gathering and checking, and qualitative assessment of between-trial differences including patient eligibility and assessment of bias have been

previously reported. ${ }^{1,11}$ No issues were identified in checking individual participant data. Medical therapy included the use of intravenous thrombolysis if clinically appropriate and 
standard follow-up care in dedicated acute stroke units. Clinical data were extracted and pooled by the study statistician. Imaging studies were de-identified and read by the independent HERMES core laboratories for baseline non-contrast Alberta Stroke Program Early CT Score (CT-ASPECTS) assessment and reprocessing of CT Perfusion (CTP) and diffusion MRI data using RAPID software (version 4.6) as previously described. ${ }^{1,11}$ All participants provided written consent and each study was approved by the local ethics board.

\section{Data analysis}

Patients presenting with LHI were defined on pre-treatment imaging as having an ischemic core volume of 80-300ml on diffusion MRI or CTP using an apparent diffusion coefficient threshold $<620 \mathrm{~mm}^{2} / \mathrm{s}$ and a relative cerebral blood flow $<30 \%$ respectively, or CT-ASPECTS $\leq 5$ on unenhanced-CT if CTP or DWI was not performed. ${ }^{3,19,20}$ Patients with core volume $80-300 \mathrm{ml}$ were included, and excluded if $<80 \mathrm{ml}$ irrespective of ASPECTS.

Reperfusion was assessed on digital subtraction angiograms in patients undergoing EVT. An expanded thrombolysis in cerebral infarction score of $2 b, 2 c$ or 3 was considered successful reperfusion. ${ }^{21}$ Follow-up Infarct Volume (FIV) was calculated using validated imaging software based on planimetry after manual segmentation as previously. ${ }^{22}$ Hemorrhagic transformation was scored according to the European Cooperative Acute Stroke Study II classification by two neurologists blinded to the clinical data $(\mathrm{BC}, \mathrm{FN})$.

The primary analysis determined factors associated with cerebral edema, as assessed by the maximal anatomical deviation of midline cerebral structures in the axial plane. This midline shift (MLS) was measured in millimetres on all available follow-up scans between 1 to 14 days measured by a neurologist (FN) blinded to clinical data. In patients who had more than one follow-up scans, the scan with the highest MLS was analysed. MLS measurements were 
independently assessed in $20 \%$ of the study cohort (38 consecutive subjects) by a second neurologist (BC) and found to have excellent interrater agreement (intraclass absolute correlation $=0.98,95 \%$ CI 0.97-0.99). Follow-up imaging performed after neurosurgical procedures was excluded from analyses. Associations with MLS were assessed by Mann-Whitney U test and repeated measures linear regression. Spearman's rho was used to assess correlation.

The secondary analysis evaluated the association between cerebral edema and functional outcome with the end point being functional improvement defined as $\geq 1$ point reduction in modified Rankin scale (mRS) at 90-days expressed as an unadjusted and adjusted common odds ratio (cOR) from ordinal logistic regression.

Finally, we tested the association between pre-treatment core volume and cerebral edema in an exploratory analysis using DWI and CTP core volume at baseline after excluding patients qualifying for LHI based on CT-ASPECTS alone. To further examine the effect of pre-treatment core volume across its entire range, we expanded the study cohort to include patients who presented with smaller ischemic core at baseline but developed LHI at 24-hours on follow-up DWI (>80ml FIV) or CT (CT-ASPECTS $\leq 5)$. Locally Estimated Scatterplot Smoothing (LOESS) regression analysis was performed in this analysis to examine the potentially variable and bimodal relationship between MLS with baseline core volume and between MLS with CTASPECTS, and to examine whether treatment modality influenced these relationships. LOESS is a non-parametric regression method to model complex non-linear relationships by assigning a weighted function to each data point which is then scatter-plotted to allow visualization of the entire dataset for hypothesis generation.

Mixed-effects modelling was used for all regression analyses and adjusted for four predetermined variables: age, pre-treatment National Institute of Health Stroke Score (NIHSS), 
baseline glucose level, and timing of follow-up up scan expressed in number of days after onset of stroke symptoms. Pre-specified interaction between MLS and reperfusion was tested in the primary analysis. Pre-specified sensitivity analyses were performed for the use of intravenous thrombolysis, whether follow-up scans were performed within or after 48-hours, baseline ischemic core volume and ASPECTS. Parenchymal hematoma (Type 1 and 2) were included in the primary analysis but adjusted in sensitivity analysis. Further sensitivity analyses were performed for differences in baseline characteristics between the treatment groups and imaging inclusion criteria (Core volume $>80 \mathrm{mls}$ vs CT-ASPECTS $\leq 5$ ).

Risk of bias was assessed using the Cochrane tool and was low overall as previously reported. ${ }^{11}$ Statistical analyses were done with SAS (version 9.4).

\section{$\underline{\text { Results }}$}

Among 1764 patients included in seven studies, 99 did not have follow-up imaging. Of the remaining 1665 patients, $177(10.6 \%)$ with LHI at baseline were analysed in the primary and secondary analysis in which patients undergoing EVT $(n=85,48.0 \%)$ were compared to patients receiving non-endovascular medical treatment $(n=92,52.0 \%)$ (Table 1). Successful reperfusion was achieved in 62/85 patients undergoing EVT (72.9\%). Patients who were randomized to EVT had lower median pre-treatment ischemic core volume, higher ASPECTS and a shorter timefrom-onset-to-presentation (Table 1). The majority of patients were defined as LHI based on CTASPECTS $(n=91,51 \%)$, followed by DWI $(n=51,29 \%)$ and CTP $(n=35,20 \%)$. Parenchymal hematoma occurred in 17 patients $(9.6 \%)$. Neurosurgical interventions for management of mass effect were performed in 10 patients ( $n=9$ decompressive hemicraniectomy; $n=1$ external ventricular drainage). More than one follow-up scan was performed in 44 patients. Overall, MLS was measured on 230 follow-up scans. 
In the primary analysis, cerebral edema as measured by median MLS on follow-up imaging (median time-from-onset 28.3 hours) was not significantly different between treatment groups (EVT 5.0[IQR2.8-8.6] vs. medical therapy 5.5[IQR3.1-8.1]mm,p=0.97). Malignant infarction ( $>5 \mathrm{~mm}$ MLS or requiring decompressive craniectomy) also occurred in a similar proportion (42/85[49.4\%] EVT vs.50/92[54.3\%]) medical therapy,p=0.51). ${ }^{23}$ In multivariable linear regression, higher baseline NIHSS was associated with increased MLS $(\beta=0.25[95 \%$ CI 0.10 0.40]) but treatment modality was not ( $\beta=-0.27$,[95\%CI-1.52-0.98], Supplemental Material). Sensitivity analyses for pre-specified variables (intravenous thrombolysis, parenchymal hematoma, follow-up scans timing, baseline ischemic core volume, ASPECTS), baseline differences between treatment groups (sex, time from symptom onset to presentation) and different imaging inclusion criteria yielded similar results (Supplemental Material). Among patients treated with EVT, reperfusion ( $\beta=-0.78$,[95\%CI-3.07-1.50]), and onset-to-reperfusion time $(\beta=0.70,[95 \%$ CI-0.04-1.43]) were not significantly associated with MLS.

In the secondary analysis, a favorable functional outcome (defined as mRS 0-2) was achieved in 27/177 patients (EVT $18.8 \%$ vs. $12.1 \%$ Control,p=0.22). MLS was independently associated with worse functional outcome ( $\mathrm{cOR}=0.82,[95 \% \mathrm{CI} 0.77-0.89])$ after adjusting for the pre-determined variables age, NIHSS, serum glucose, time-to-follow-up-imaging, and EVT (Supplemental Material).

EVT $(\mathrm{cOR}=2.30,[95 \% \mathrm{CI} 1.32-4.00])$ was significantly associated with functional improvement. Reperfusion, when substituted for EVT in the multivariable model, was also significantly associated with functional improvement (cOR=4.73,[95\%CI1.66-13.52]). There was a significant interaction effect between MLS and reperfusion ( $\mathrm{p}=0.038)$ whereby the benefit of reperfusion reduced as MLS increased. 
The median FIV in the study cohort was 218.4mL (IQR108.0-307.9), with no significant difference between the EVT group (208.4ml[IQR89.2-289.7]) and the medical management group (223.2mL[IQR140.7-347.1],p=0.305). MLS significantly correlated with FIV $(\sigma=0.57, \mathrm{p}<0.0001)$

In an exploratory analysis of the association between pre-treatment ischemic core volume and cerebral edema, pre-treatment ischemic core volume was measured on baseline DWI and CTP in 86/177 patients after excluding patients who qualified for LHI based on CT-ASPECTS alone.

Pre-treatment core volume was independently associated with MLS ( $\beta=0.02,[95 \% \mathrm{CI} 0.00-0.04])$ in multivariable analysis within this subgroup (Supplemental Material).

Further analysis was performed in an expanded study cohort that included an additional 741 patients who presented with smaller ischemic core at baseline but subsequently developed LHI at 24-hours. The associations between EVT and MLS, and between MLS and functional outcome in this expanded cohort were consistent with the primary study cohort of 177 patients presenting with LHI (Supplemental Material).

On a LOESS scatterplot with pre-treatment ischemic core volume (DWI or CTP) on the X-axis and MLS on the $\mathrm{y}$-axis ( $\mathrm{n}=641$ patients), cerebral edema as measured by MLS was similar between patients treated with EVT and medical therapy throughout the lower ranges of core volume but was greater in patients treated with EVT when core volume exceeded $130 \mathrm{ml}$ on visual inspection (Figure 1). A similar pattern was observed when CT-ASPECTS was used as a surrogate for ischemic core volume ( $\mathrm{n}=910$ patients) with greater MLS in patients treated with EVT who had pre-treatment CT-ASPECTS $\leq 3$ (Supplemental Material).

Based on the visual interpretation of the LOESS scatterplots, patients presenting with core volume $>130 \mathrm{ml}$ or CT-ASPECTS $\leq 3$ ( $\mathrm{n}=76$ MLS measurements) were pooled for further post-hoc 
analysis. In this subgroup of very large infarcts, EVT was associated with greater MLS (8.6mm[IQR5.5-13.8] vs.6.0mm[IQR3.3-8.4] medical therapy, $\mathrm{p}=0.025)$ in univariable analysis and after adjustment for age and NIHSS ( $\beta=2.76[95 \%$ CI-0.33-5.20]). No significant difference in functional outcome between EVT and control groups was observed in this very large infarct subgroup (cOR=1.75[95\%CI0.62-4.89]). In comparison, in the remaining subgroup of patients present presenting with core volume $80-130 \mathrm{ml}$ or CT-ASPECTS 4-5 (n=154 MLS measurements), EVT was not associated with MLS (4.1mm[IQR 2.2-6.9] vs. 4.6mm[IQR2.97.8], $\mathrm{p}=0.33$ ) on univariable analysis but was associated with functional improvement $(\mathrm{cOR}=2.11[95 \% \mathrm{CI} 1.08-4.09])$ (Figure 2).

\section{$\underline{\text { Discussion }}$}

In this patient-level meta-analysis of a high-risk population for secondary injury following ischemic stroke, EVT and reperfusion were associated with improved functional outcome but not cerebral edema in patients presenting with LHI, except in a subgroup of patients presenting with very extensive established ischemia with core volumes $>130 \mathrm{mls}$ or CT-ASPECTS $\leq 3$. Clinical severity and the extent of irreversible ischemic tissue at baseline were associated with cerebral edema development. Cerebral edema was associated with worse outcome and represents a potential therapeutic target to improve the clinical outcome of patients with LHI.

Our findings provide fresh insights into a topic that has yielded conflicting results between preclinical and clinical data. In preclinical animal models, transient MCA occlusion paradoxically led to greater infarct volume, brain water content and blood-brain barrier disruption compared to permanent occlusion. ${ }^{6}$ In contrast, clinical studies demonstrated an association between reperfusion and reduced cerebral edema. In a secondary analysis of the MRCLEAN trial, successful endovascular reperfusion was associated with less MLS. ${ }^{8}$ In the SITS- 
International Stroke Treatment Registry, recanalization was found to be associated with less edema on qualitative visual assessment. ${ }^{10}$ Similarly, a retrospective observational study (Broocks et al) showed less tissue water uptake in the ischemic lesion in patients with successful recanalisation. ${ }^{9}$

There are several notable differences in methodology between the current analysis and these studies. We restricted our primary study cohort to patients with evidence of LHI at baseline in whom there is more non-salvageable tissue and less penumbral tissue to mediate the benefit of infarct growth reduction. Although Broocks only included patients presenting with low ASPECTS, the substantially smaller FIV (median 120.6ml vs.218.0ml) and lower MLS (mean $3.2 \mathrm{ml}$ vs. $6.2 \mathrm{ml}$ ) compared to our cohort indicates a less severely affected group with less extensive pre-treatment infarction. EVT was not randomized in Broocks and is therefore susceptible to selection bias and confounding factors swaying clinicians' treatment decision. Further, cerebral edema was measured using tissue Net Water Uptake (NWU) instead of MLS. Experimental and clinical data suggest pathological tissue water uptake at tissue level may not inevitably lead to macroscopic swelling with anatomical distortion. At tissue level, ionic edema has been shown to be partially reversible in animal models.$^{24}$ In a post-hoc analysis of the GAMES-RP study, MLS was a stronger predictor of malignant edema and the main driver of clinical deterioration when compared to NWU. ${ }^{25}$ Finally, NWU is derived from CT Hounsfield units and has been validated on admission CT in acute ischemic stroke. It is unclear how posttreatment intracranial hemorrhage and iodine-based contrast staining after EVT influence posttreatment NWU measurements.

Our finding that the edema development in response to reperfusion may be dependent on the physiological state of the tissue provides a potential explanation for the reported discrepancy among previous studies. A bimodal response to reperfusion was first described in preclinical 
studies comparing the effect of reperfusion across animals subjected to different severity of ischemia. Reperfusion after mild to moderate ischemia was associated with less edema . However, after severe ischemia from which tissue was unlikely to survive even with reperfusion, edema increased after reperfusion as this supplied blood flow and water content to alreadyinfarcted tissue. ${ }^{5,7}$ In concordance, our exploratory analysis suggests that EVT increased mass effect among patients with substantial amount of irreversible tissue (>130ml/ASPECTS $\leq 3)$, while there was no association between EVT and edema when the study population included patients with less ischemic core and likely more salvageable tissue. Similarly, it is plausible that pre-clinical studies used MCA occlusion models that typically generated established infarction in the majority of the MCA territory rapidly and therefore demonstrated a detrimental effect of reperfusion, while clinical studies predominantly included patients who had relatively small ischemic core volumes, thus facilitating the observed beneficial effect of reperfusion on edema. Our findings may therefore be complementary, rather than contradictory, to the existing clinical data by demonstrating the alternate spectrum of the bimodal response of edema formation to reperfusion.

Our analysis adds further evidence supporting the safety and benefit of EVT in patients presenting with large ischemic core. We showed EVT and reperfusion to be associated with functional improvement, with the treatment benefit extending to patients presenting with core volume up to $130 \mathrm{ml}$ or CT-ASPECTS $\leq 3$. Our results suggest the benefit of reperfusion in patients with large ischemic core extends beyond reducing mass effect. Despite presenting with substantial established infarct, salvaging the remaining penumbral tissue may still be clinically meaningful. Partial tissue salvage even within regions considered as infarcted may also be present. In a mediation analysis of the HERMES dataset, FIV reduction was found to explain only $12 \%$ of the benefit of EVT, suggesting patients had better outcomes after EVT despite 
largely similar FIV. ${ }^{22}$ This hypothesis is further supported by recent clinical data demonstrating intra-lesional heterogeneity and a gradient of tissue injury proportional to the severity of the initial ischemia within the FIV lesion. ${ }^{26}$

EVT was not significantly associated with clinical benefit and may be detrimental by exacerbating edema beyond a core volume $>130 \mathrm{ml}$ or CT-ASPECTS $\leq 3$. However, the current dataset was not powered to evaluate this small subpopulation and our findings in this subanalysis can only be interpreted as exploratory. Several ongoing trials investigating the role of thrombectomy in patients with large core, including TENSION[NCT03094715], TESLA[NCT03805308], SELECT-2[NCT03876457] and, IN EXTREMIS

LASTE[NCT03811769] are expected to provide further insights. Based on this analysis, the interpretation of large core reperfusion trials may need to be considered in the context of baseline infarction volumes, with attention to potential differences in treatment responses between the moderate large core $(70-130 \mathrm{ml})$ and very large core $(>130 \mathrm{ml})$ strata.

Cerebral edema with mass effect as a secondary injury was independently associated with poor functional outcome. Our observations support the importance of cerebral edema as a therapeutic target in this population of patients presenting with LHI. A phase 3 randomized controlled trial CHARM(NCT02864953) is currently underway to evaluate the efficacy of intravenous glibenclamide, a SUR1-TRPM4 antagonist, for severe cerebral edema associated with LHI.

The strengths of the study are the prospective randomized allocation of reperfusion treatment and the use of objective imaging criteria with independent core laboratory adjudication for ASPECTS and automated core volume calculations for diffusion MRI and CTP. The main limitation is the relatively small study population in relation to the overall HERMES cohort due to several of the component studies excluding patients presenting with large core volume. As a 
result, our study was underpowered, even in this meta-analysis, to assess the effect of reperfusion grade and time-to-reperfusion, as well as the $>130 \mathrm{ml}$ core volume group. As the $>130 \mathrm{ml}$ core volume threshold is based on LOESS fitting within a limited sample size with large variability in MLS, further confirmatory investigation of this subgroup is warranted. Second, additional imaging beyond the first (24-hour) follow-up scan was not standardized. As mass effect usually peaks between day 2 to 4 after stroke, we may not have captured the maximum MLS in patients who remained stable and did not have clinical indications for re-imaging. Third, MLS represents a widely used, easily accessible and validated quantitative measure of the space-occupying effect of cerebral edema. In experimental stroke models, MLS has been shown to strongly correlate with absolute hemispheric water content by weight on wet-dry analysis. ${ }^{27}$ Clinically, MLS has been shown to correlate with increased intracranial pressure, malignant infarction, and poor outcome in ischemic stroke. ${ }^{8,28}$ However, MLS may be disproportionally affected by infarct topography and pre-existing cerebral atrophy. MLS may under-represent focal edema in cortical regions and may over-represent pathology in the deep structures closer to midlines. This may be improved by volumetric analysis accounting for lesion swelling volume. ${ }^{23}$ Incorporating edema assessment at the tissue level using quantitative water uptake may further enhance evaluation of cerebral edema. ${ }^{29}$ Fourth, the study included patients qualified with different imaging modalities which may contribute to heterogeneity in the study cohort. To date, there is no consensus on the imaging definition of LHI and the combined use of CTP core volume, diffusion MRI lesion volume and CT-ASPECTS was chosen as a pragmatic option given that all of these are used in the literature. Finally, the study cohort was limited to patients presenting within the early treatment time window of 6 hours from symptoms onset. Further studies are needed to validate our findings in patients presenting in the late treatment window. 
In summary, our data provide reassurance that iatrogenic exacerbation of space-occupying cerebral edema following EVT in patients is unlikely in patients presenting with LHI, particularly among patients with core volume $<130 \mathrm{ml}$ and ASPECTS $>3$. The variable edema response to reperfusion based on the physiological state of cerebral tissue provides a potential explanation for discrepancies among previous studies. Trials directed at mitigating the effects of cerebral edema offer an avenue to reduce morbidity and mortality in patients with LHI. 


\section{ACKNOWLEDGMENTS}

None

\section{FUNDING}

Medtronic provided funding but had no role in the design or analysis. Biogen provided funding and reviewed the analysis plan and manuscript.

\section{DISCLOSURES}

BCVC: Research support from National Health and Medical Research Council of Australia (GNT1043242 and GNT1035688), Royal Australasian College of Physicians, Royal Melbourne Hospital Foundation, National Heart Foundation, National Stroke Foundation of Australia, and unrestricted grant funding for the EXTEND-IA trial to the Florey Institute of Neuroscience and Mental Health from Covidien (Medtronic). CBLMM: Research support from CVON/Dutch Heart Foundation, European Commission, TWIN foundation, Dutch Health Evaluation Program and Stryker (all paid to institution) and owns stock in Nico-lab, a company that focuses on the use of artificial intelligence for medical image analysis (modest). WTK: Research grants from National Institute of Neurological Disorders and Stroke, the American Heart Association, Biogen, and NControl Therapeutics, and personal fees from Biogen and NControl Therapeutics, and has a patent pending (16/486,687 and PCT/US2018/018537) for a method and composition for treating a brain injury. BKM: Member of the steering and executive committee for the ESCAPE trial, which received support from Covidien (Medtronic), was site principal investigator for the SOCRATES trial (sponsored by AstraZeneca), has received honoraria from 
Penumbra, has a provisional patent (62/086077) for triaging systems in ischemic stroke, and has research funding from Canadian Institutes of Health Research, Heart and Stroke Foundation of Canada, Alberta Innovates-Health Solutions, and the Hotchkiss Brain Institute and the Faculty of Medicine, University of Calgary; shareholding from Circle NVI. AMD: Grant funding from Medtronic for the ESCAPE trial and personal fees from Medtronic; stock ownership from Circle NVI; patent to Circle NVI. PW: Grant funding to University of Glasgow for the PISTE trial from Medtronic and Codman as well as grants from Stroke Association (TSA 2011/06) and National Institute of Health Research (NIHR) Health Technology Assessment programme (HTA 14.08.47), grants and personal fees from Microvention Terumo and personal fees from Stryker and Codman. AD: Grant funding for REVASCAT trial and personal fees from Medtronic. SMD: Personal fees from Medtronic and Boehringer Ingelheim. SBro: Personal fees from Medtronic and the University of Calgary. KWM: Personal fees from Biogen, Bayer, for consultancy from Medtronic, personal feeds and non-financial support from Boehringer Ingelheim, personal fees from Daiichi Sankyo, Abbvie, ReNeuron. The University of Glasgow received grant support for the PISTE trial from Medtronic and Codman as well as grants from the Stroke Association (TSA 2011/06) and NIHR Health Technology Assessment programme (HTA $14 \cdot 08 \cdot 47)$. DWJD: Grants from the Dutch Heart Foundation, AngioCare BV, Medtronic/Covidien/EV3, MEDAC Gmbh/LAMEPRO, Penumbra, Top Medical/Concentric, and Stryker, and his institution received consultancy fees from Stryker, Bracco Imaging, and Servier. MG: Grants from Medtronic and Stryker, personal fees from Mentice, Medtronic, Stryker, Microvention and GE Healthcare; a patent systems and methods for diagnosing strokes (PCT/CA2013/000761) licensed to GE Healthcare. JLS: Unpaid site investigator in multicentre trials sponsored by Covidien, Medtronic/Abbott, Stryker, and Neuravi/Abbott, for which the University of California received payments on the basis of clinical trial contracts for the number of subjects enrolled; reports 
receiving contracted hourly payments and travel reimbursement from Covidien, Medtronic/Abbott, Stryker, and Neuravi/Abbott, and stock options from Rapid Medical, for service on Trial Steering Committees, advising on rigorous trial design and conduct. The University of California has patent rights in retrieval devices for stroke. TGJ: Viz.ai; Personal fees for consultancy from Contego Medical, Cerenovus, Codman Neurovascular and Neuravi, holds stock in Silk Road, Anaconda, Route 92, FreeOx Biotech, Blockade and Corindus; grants from Metronic; has acted as an unpaid consultant to Stryker as principal investigator of the DAWN trial; has acted as investor/advisor to Methinks. MDH: Medtronic LLC during the conduct of the study; grants from NoNO Inc, Boehringer Ingelheim, and Biogen Inc outside the submitted work; a patent to US Patent office Number: 62/086,077 issued and licensed; Stock owner in Pure Web Incorporated and CircleNVI, director of the Canadian Federation of Neurological Sciences, the Canadian Stroke Consortium and Circle NeuroVascular Inc., public grant support to the University of Calgary from Alberta Innovates Health Solutions, CIHR, Heart \& Stroke Foundation of Canada, National Institutes of Neurological Disorders and Stroke. PJM: Unrestricted research grants to his institution from Codman Johnson and Johnson, Medtronic, and Stryker and has served as an unpaid consultant to Codman Johnson and Johnson. MJW: Employee and shareholder of Biogen. All other authors declare no competing interests. KS: grants from Biogen during the conduct of the study. DL: Cerenovus, Genentech, Metrodnic, Stryker.

\section{SUPPLEMNTAL MATERIALS}

Online Figures I-VI

Online Figure I-II 


\section{REFERENCES}

1. Roman LS, Menon BK, Blasco J, Hernandez-Perez M, Davalos A, Majoie C, Campbell BCV, Guillemin F, Lingsma H, Anxionnat R, et al. Imaging features and safety and efficacy of endovascular stroke treatment: a meta-analysis of individual patient-level data. Lancet Neurol 2018; 17(10): 895-904.

2. Vahedi K, Hofmeijer J, Juettler E, Vicaut E, George B, Algra A, Amelink GJ, Schmiedeck P, Schwab S, Rothwell PM, et al. Early decompressive surgery in malignant infarction of the middle cerebral artery: a pooled analysis of three randomised controlled trials. Lancet Neurol 2007; 6(3): 215-22.

3. Liebeskind DS, Juttler E, Shapovalov Y, Yegin A, Landen J, Jauch EC. Cerebral Edema Associated With Large Hemispheric Infarction. Stroke 2019; 50(9): 2619-25.

4. Berrouschot J, Sterker M, Bettin S, Koster J, Schneider D. Mortality of space-occupying ('malignant') middle cerebral artery infarction under conservative intensive care. Intensive Care Med 1998; 24(6): 620-3.

5. Iannotti F, Hoff J. Ischemic brain edema with and without reperfusion: an experimental study in gerbils. Stroke $1983 ; \mathbf{1 4}(4)$ : 562-7.

6. Yang GY, Betz AL. Reperfusion-induced injury to the blood-brain barrier after middle cerebral artery occlusion in rats. Stroke 1994; 25(8): 1658-64; discussion 64-5.

7. Bell BA, Symon L, Branston NM. CBF and time thresholds for the formation of ischemic cerebral edema, and effect of reperfusion in baboons. J Neurosurg 1985; 62(1): 31-41.

8. Kimberly WT, Dutra BG, Boers AMM, Alves H, Berkhemer OA, van den Berg L, Sheth KN, Roos Y, van der Lugt A, Beenen LFM, et al. Association of Reperfusion With Brain Edema in Patients With Acute Ischemic Stroke: A Secondary Analysis of the MR CLEAN Trial. JAMA Neurol 2018; 75(4): 453-61. 
9. Broocks G, Hanning U, Flottmann F, Schonfeld M, Faizy TD, Sporns P, Baumgart M, Leischner H, Schon G, Minnerup J, et al. Clinical benefit of thrombectomy in stroke patients with low ASPECTS is mediated by oedema reduction. Brain 2019; 142(5): 1399-407.

10. Thoren M, Dixit A, Escudero-Martinez I, Gdovinova Z, Klecka L, Rand VM, Toni D, Vilionskis A, Wahlgren N, Ahmed N. Effect of Recanalization on Cerebral Edema in Ischemic Stroke Treated With Thrombolysis and/or Endovascular Therapy. Stroke 2020; 51(1): 216-23.

11. Campbell BCV, Majoie C, Albers GW, Menon BK, Yassi N, Sharma G, van Zwam WH, van Oostenbrugge RJ, Demchuk AM, Guillemin F, et al. Penumbral imaging and functional outcome in patients with anterior circulation ischaemic stroke treated with endovascular thrombectomy versus medical therapy: a meta-analysis of individual patient-level data. Lancet Neurol 2019; 18(1): 46-55.

12. Berkhemer OA, Fransen PS, Beumer D, van den Berg LA, Lingsma HF, Yoo AJ, Schonewille WJ, Vos JA, Nederkoorn PJ, Wermer MJ, et al. A randomized trial of intraarterial treatment for acute ischemic stroke. N Engl J Med 2015; 372(1): 11-20.

13. Campbell BC, Mitchell PJ, Kleinig TJ, Dewey HM, Churilov L, Yassi N, Yan B, Dowling RJ, Parsons MW, Oxley TJ, et al. Endovascular therapy for ischemic stroke with perfusion-imaging selection. N Engl J Med 2015; 372(11): 1009-18.

14. Goyal M, Demchuk AM, Menon BK, Eesa M, Rempel JL, Thornton J, Roy D, Jovin TG, Willinsky RA, Sapkota BL, et al. Randomized assessment of rapid endovascular treatment of ischemic stroke. N Engl J Med 2015; 372(11): 1019-30.

15. Saver JL, Goyal M, Bonafe A, Diener HC, Levy EI, Pereira VM, Albers GW, Cognard C, Cohen DJ, Hacke W, et al. Stent-retriever thrombectomy after intravenous t-PA vs. t-PA alone in stroke. N Engl J Med 2015; 372(24): 2285-95. 
16. Jovin TG, Chamorro A, Cobo E, de Miquel MA, Molina CA, Rovira A, San Roman L, Serena J, Abilleira S, Ribo M, et al. Thrombectomy within 8 hours after symptom onset in ischemic stroke. N Engl J Med 2015; 372(24): 2296-306.

17. Muir KW, Ford GA, Messow CM, Ford I, Murray A, Clifton A, Brown MM, Madigan J, Lenthall R, Robertson F, et al. Endovascular therapy for acute ischaemic stroke: the Pragmatic Ischaemic Stroke Thrombectomy Evaluation (PISTE) randomised, controlled trial. J Neurol Neurosurg Psychiatry 2017; 88(1): 38-44.

18. Bracard S, Ducrocq X, Mas JL, Soudant M, Oppenheim C, Moulin T, Guillemin F, investigators T. Mechanical thrombectomy after intravenous alteplase versus alteplase alone after stroke (THRACE): a randomised controlled trial. Lancet Neurol 2016; 15(11): 1138-47. 19. Thomalla GJ, Kucinski T, Schoder V, Fiehler J, Knab R, Zeumer H, Weiller C, Rother J. Prediction of malignant middle cerebral artery infarction by early perfusion- and diffusionweighted magnetic resonance imaging. Stroke 2003; 34(8): 1892-9.

20. Schroder J, Cheng B, Ebinger M, Kohrmann M, Wu O, Kang DW, Liebeskind DS, Tourdias T, Singer OC, Christensen S, et al. Validity of acute stroke lesion volume estimation by diffusion-weighted imaging-Alberta Stroke Program Early Computed Tomographic Score depends on lesion location in 496 patients with middle cerebral artery stroke. Stroke 2014; 45(12): 3583-8.

21. Liebeskind DS, Bracard S, Guillemin F, Jahan R, Jovin TG, Majoie CB, Mitchell PJ, van der Lugt A, Menon BK, San Roman L, et al. eTICI reperfusion: defining success in endovascular stroke therapy. J Neurointerv Surg 2019; 11(5): 433-8.

22. Boers AMM, Jansen IGH, Brown S, Lingsma HF, Beenen LFM, Devlin TG, Roman LS, Heo JH, Ribo M, Almekhlafi MA, et al. Mediation of the Relationship Between Endovascular 
Therapy and Functional Outcome by Follow-up Infarct Volume in Patients With Acute Ischemic Stroke. JAMA Neurol 2019; 76(2): 194-202.

23. Kauw F, Bennink E, de Jong H, Kappelle LJ, Horsch AD, Velthuis BK, Dankbaar JW, Investigators D, follows Diaa. Intracranial Cerebrospinal Fluid Volume as a Predictor of Malignant Middle Cerebral Artery Infarction. Stroke 2019; 50(6):STROKEAHA119024882.

24. Ito U, Ohno K, Nakamura R, Suganuma F, Inaba Y. Brain edema during ischemia and after restoration of blood flow. Measurement of water, sodium, potassium content and plasma protein permeability. Stroke 1979; 10(5): 542-7.

25. Vorasayan P, Bevers MB, Beslow LA, Sze G, Molyneaux BJ, Hinson HE, Simard JM, von Kummer R, Sheth KN, Kimberly WT. Intravenous Glibenclamide Reduces Lesional Water Uptake in Large Hemispheric Infarction. Stroke 2019; 50(11): 3021-7.

26. Ng F, Venkatraman V, Parsons M, Bivard A, Sharma G, Churilov L, Desmond P, Davis SM, Yassi N, Campbell B. Gradient of Tissue Injury after Stroke: Rethinking the Infarct versus Noninfarcted Dichotomy. Cerebrovasc Dis 2020; 49(1): 32-8.

27. Walberer M, Blaes F, Stolz E, Muller C, Schoenburg M, Tschernatsch M, Bachmann G, Gerriets T. Midline-shift corresponds to the amount of brain edema early after hemispheric stroke--an MRI study in rats. J Neurosurg Anesthesiol 2007; 19(2): 105-10.

28. Liao CC, Chen YF, Xiao F. Brain Midline Shift Measurement and Its Automation: A Review of Techniques and Algorithms. Int J Biomed Imaging 2018; 2018: 4303161.

29. Nawabi J, Flottmann F, Hanning U, Bechstein M, Schon G, Kemmling A, Fiehler J, Broocks G. Futile Recanalization With Poor Clinical Outcome Is Associated With Increased Edema Volume After Ischemic Stroke. Invest Radiol 2019; 54(5): 282-7. 


\section{FIGURE LEGENDS}

Figure 1- Scatterplot of pre-treatment core volume with midline shift

Scatterplot with Locally Estimated Scatterplot Smoothing of pretreatment core volume with MLS ( $\mathrm{n}=641)$ in patients with LHI at baseline and at 24hours.

Figure 2 - Comparison of cerebral edema by treatment

Boxplot of cerebral edema expressed in midline shift between treatment modality stratified by patient subgroups. There were no differences in midline shift between the thrombectomy (EVT) and control in the overall analysis $(\mathrm{p}=0.97)$, and in the $80-130 \mathrm{ml}$ or CT-ASPECTS $4-5$ subgroup $(\mathrm{p}=0.33)$ on univariable analysis. In comparison, patients with EVT had increased midline shift in the $80-130 \mathrm{ml}$ or CT-ASPECTS $\leq 3$ subgroup $(\mathrm{p}=0.025)$. 
Table 1 Baseline clinical and imaging characteristics of all patients presenting with large hemispheric infarction

\begin{tabular}{|c|c|c|c|c|}
\hline & \multicolumn{3}{|c|}{ Large Hemispheric Infarction $(n=177)$} & \multirow{2}{*}{$\begin{array}{l}\text { All Participants } \\
\qquad(n=1764)\end{array}$} \\
\hline & $\begin{array}{l}\text { Medical therapy } \\
\text { group }(n=92)\end{array}$ & $\begin{array}{l}\text { Endovascular } \\
\text { thrombectomy group } \\
(n=85)\end{array}$ & $\mathrm{P}$ value & \\
\hline Age, years & $63.6 \pm 13.3$ & $62.0 \pm 12.9$ & 0.40 & $65.6(13.5)$ \\
\hline Female sex & $43.5 \%(40)$ & $24.7 \%(21)$ & 0.011 & $835(47 \%)$ \\
\hline Baseline NIHSS & $20(17-23)$ & $19(17-22)$ & 0.11 & $17(13-21)$ \\
\hline $\begin{array}{l}\text { Onset-to- } \\
\text { emergency- } \\
\text { department, min }\end{array}$ & $105.0(65.0,170.0)$ & $95.0(55.8,151.3)$ & 0.003 & $105(60-180)$ \\
\hline $\begin{array}{l}\text { Baseline (pre- } \\
\text { treatment) } \\
\text { ischemic core } \\
\text { volume, ml }\end{array}$ & $\begin{array}{c}124.0(92.8- \\
157.1)\end{array}$ & $97.1(81.6-118.5)$ & 0.005 & N/A \\
\hline $\begin{array}{l}\text { Baseline (pre- } \\
\text { treatment) } \\
\text { ASPECTS }\end{array}$ & $4(3-5)$ & $5(4-5)$ & 0.027 & $8(7-9)$ \\
\hline
\end{tabular}

Data are mean (SD), median (IQR), or n (\%). ASPECTS = Alberta Stroke Program Early CT

score. N/A=not applicable. NIHSS = National Institutes of Health Stroke Scale 